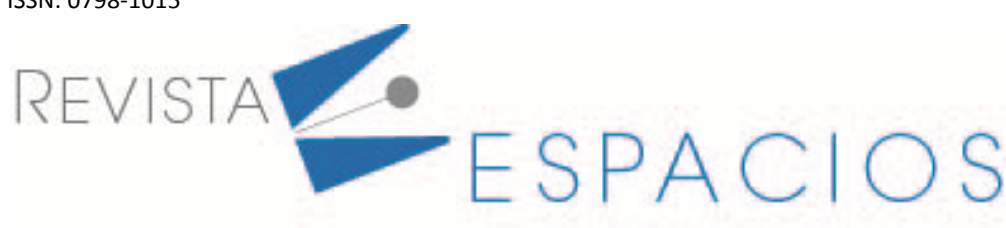

\title{
Etapas de la reforma curricular en programas de ciencias administrativas para la formación en sostenibilidad
}

\section{Stages of curriculum reform in management science programs for sustainability training.}

\author{
PAREDES-CHACÍN, Ana J. ${ }^{1}$ \\ RODRÍGUEZ SÁNCHEZ, María del P. ${ }^{2}$ \\ PEREA CASTILLO, María C. ${ }^{3}$
}

\begin{abstract}
Resumen
Sistematizar las etapas de la reforma curricular en los programas de ciencias administrativas para la formación en sostenibilidad fundamentan el objetivo del artículo. El método establece cinco etapas, estructuradas desde una investigación analítica-documental, propositiva. Los resultados consideran los actores que demandan la renovación del currículo: universidad-empresa-Estado-sociedad. Se concluye sobre la importancia de viabilizar el diseño presentado desde un enfoque integral, como estrategia de fortalecimiento de la formación de profesionales en ciencias administrativas con competencias para impulsar el desarrollo sostenible.

Palabras clave: reforma curricular; educación superior; formación en sostenibilidad; programas académicos; ciencias sociales.

Abstract

Systematizing the stages of the curricular reform in management sciences programs for sustainability training underping the objective of the article. The method establishes five stages, structured from an analytical-documentary, propositional research. The results consider the actors that demand the renewal of the curriculum: university-business-states-society. It is concluded, on the importance of making the design presented viable from an integral approach, as a strategy to strengthen the formation of professionals in administrative sciences with competencies to promote sustainable development.

key words: curricular reform, higher education, sustainability training, academic programs, social sciences.
\end{abstract}

\section{Introducción}

Entre las principales iniciativas que se impulsan desde las instituciones de educación superior en la ultima década se destacan las formas de dinamizar el proceso de aprendizaje, y con este las estrategias de formación en la cual el tema asociado con la reforma curricular, se considera como una de las acciones que requieren de un permanente proceso de evaluación y adaptación para responder a las realidades que demanda una sociedad en constante transformación. Como parte de la gestión académica para garantizar el mejoramiento continuo en la educación superior, es necesario determinar las capacidades de las instituciones de educación superior,

\footnotetext{
${ }^{1}$ Coordinadora del Grupo de Investigación en Ciencias Administrativa. Coordinadora de la Línea de Investigación Gestión de Organizaciones Sostenibles. Departamento de Administración y Finanzas. Universidad Autónoma de Occidente. ajparedes@uao.edu.co (autora de correspondencia)

2 Profesora a Tiempo completo. Departamento de Administración y Finanzas.Universidad Autónoma de Occidente. mprodriguez@uao.edu.co

${ }^{3}$ Profesora a Tiempo completo. Departamento de Administración y Finanzas.Universidad Autónoma de Occidente. mcperea@uao.edu.co
} 
en adelante IES, con el fin de promover la calidad de los recursos que soportan el quehacer educativo y por ende los programas académicos, en este caso los asociados con las ciencias administrativas, proyectado su alcance desde el contexto latinoamericano.

Entre los factores que fundamentan la reforma curricular, se asocian la proyección hacia la denominada calidad educativa, la cual amerita responder a la formación de profesionales cuyo perfil esté asociado con las transformaciones sociales, económicas y productivas de una sociedad que interactúa en mercados de bienes y servicios que compiten en un orden global.

La Organización de las Naciones Unidas para la Educación, la Ciencia y la Cultura, (UNESCO 2006, 2008), entre sus lineamientos para respaldar la calidad educativa, plantea acuerdos desde el punto de vista teórico y de generación de valor utilizado en los procesos formativos, declaración que proyecta la relevancia de la calidad educativa. Sin embargo, el concepto como tal es complejo, por sus diversas definiciones, para lo cual se requiere de una comprensión precisa que permita su operatividad en el campo de actuación. Al respecto, la mayoría de las reformas académicas, se asocian al concepto de resultados, centrados en una educación que contribuya al aprendizaje permanente, asociado con las competencias y capacidades apropiadas para un desarrollo a lo largo de la vida. A partir de lo expuesto, se requiere garantizar, tanto la calidad de la educación superior, como generar capacidades en las IES, mediadas por los organismos internacionales, nacionales y regionales, que promueven los criterios de reconocimiento para atribuir al reconocimiento de acreditación institucional.

Brunner y Pedraja-Rejas (2017) exponen que en el entorno de la educación superior, se consolidan esquemas públicos de evaluación sobre la calidad de los procesos que se impulsan desde estas instituciones. Desde esta perpectiva, para promover el fortalecimiento del subsistema educativo, se requiere de una planificación y renovada visión sobre la formación basada en fundamentos que se afianzan mediante la interacción entre la teoría y la práctica. De esta forma, se viabilizan parte de los esquemas que contribuyen con la asertividad del proceso de aprendizaje, para lo cual se requiere, de efectivas formas de transferir y promover la apropiación del conocimiento como base diferenciadora que proyecta la pertinencia de programas académicos direccionados hacia la transformación del individuo y por ende de la sociedad.

Ante lo descrito, y mediante el direccionamiento del estudio, se asocia la lógica centrada en el fortalecimiento de una nueva realidad permeada por los principios del desarrollo sostenible, el cual, bajo el enfoque de las ciencias administrativas, se reafirma como un aspecto necesario, que requiere enmarcarse desde la transversalidad de los procesos formativos en la educación superior y ser proyectados mediante una visión de corresponsabilidad y de calidad educativa en la que interactúan universidad-empresa-Estado-sociedad.

La concepción de la calidad, según la Organización para la Cooperación y el Desarrollo Económicos, OECD (1996) y la Organización de las Naciones Unidas para la Educación, la Ciencia y la Cultura, UNESCO (2009) bajo los esquemas públicos, ha de viabilizarse a partir de iniciativas centradas en resultados que garanticen la pertinencia de los procesos formativos en los cuales, el currículo dinamiza y logra estructurar, el quehacer formativo en la educación superior y a su vez, se proyecta como el componente que direcciona la gestión académica para el desarrollo de competencias y capacidades en las personas para su desarrollo a lo largo de la vida.

En función de ello, se define como objetivo sistematizar las etapas de la reforma curricular en programas de ciencias administrativas para la formación en sostenibilidad. El desarrollo del estudio parte de la fundamentación teórica que rige los principios de la reforma curricular desde las ciencias administrativas, sus bases permiten la estructura de las etapas asociadas a la formación en sostenibilidad, lo cual se fundamenta mediante el enfoque metodológico establecido como base para la definición de las propuestas de cada etapa, así como la posterior disertación y aspectos concluyentes. 


\subsection{Reforma curricular desde las instituciones de educación superior}

La reforma curricular, parte de la capacidad de las IES de impulsar estándares que permiten viabilizar de forma asertiva, el análisis y desarrollo de la gestión centrada en: a) revisión de diseños programáticos; b) la interrelación de las áreas temáticas; c) la adopción de prácticas pedagógicas y didácticas; d) la visión transdisciplinaria de la investigación; e) estrategias para fomentar la creatividad, innovación y el emprendimiento; f) capacidad de interacción con entornos sociales y organizacionales como escenarios para la práctica de los saberes.

Al respecto, Muñoz (2017) afirma que existen evidencias en Centroamérica, las cuales sugieren que países más abiertos al exterior, son más proclives a adoptar diversas políticas educativas, entre estas las reformas curriculares. Proceso académico, considerado como un aspecto que amerita dar respuesta al desenvolvimiento y desarrollo de una sociedad en constante transformación. Es así, que promover la calidad educativa desde las IES, requiere del conocimiento de los factores estratégicos que fundamentan los procesos de reformas curriculares. Para la Oficina Regional de Educación de la UNESCO para América Latina y el Caribe (OREALC/UNESCO Santiago 2007) lograr el fortalecimiento de los procesos de reformas curriculares, es una primera aproximación de lo que es calidad de la educación, al afirmar, que la calidad educativa es un medio para que el ser humano se desarrolle plenamente como tal, crezca y se fortalezca como persona y especie que contribuye al desarrollo de la sociedad, transmitiendo y compartiendo sus valores y su cultura.

El currículo para Qu, et al. (2020), responde al sistema de planes de estudios, se compone de cursos establecidos por la especialidad según la disposición y la combinación del contenido y el proceso de enseñanza, que es un estado relativamente complejo y dinámico, asimismo, constituye el núcleo de la formación de talentos profesionales. Mientras que para Ratcliff (1997) es el instrumento de soporte académico, sistematizado a través del cual, se estructura tanto el proceso, como el contenido y la esencia de un programa educacional.

Entre las bases de su diseño, se destaca el propósito, el método y las técnicas didácticas o metodologías activas, para ser impartido, así como la evaluación de los aprendizajes y experiencias educativas. La organización de un currículo se define por la filosofía educativa, la estructura y el contenido del conocimiento que se imparte, y por el contexto y clima institucional.

Silva (2015) destaca que en el campo curricular confluyen las visiones y los valores de una sociedad. En la concepción del currículo existen perspectivas de reproducción social, hasta propuestas alternativas a la lógica del mercado. En función de ello, los criterios pedagógicos y didácticos, se conciben como parte de la complementariedad estratégica para generar las bases que proyectan excelencia académica.

La viabilidad de la excelencia académica parte de las capacidades de los docentes e investigadores, quienes apoyados por recursos determinados por lo cognitivo, tecnológicos y de información, impulsan modelos emergentes de formación, los cuales ameritan ser estructurados de forma sistémica en el proceso de formación de profesionales con competencias permanentes y capacidades para integrarse en el mercado laboral competitivo, por lo cual, el repensar la pertinencia de la educación superior, es un tema inaplazable como estrategia para afianzar el proceso de formación basado en la sostenibilidad, en donde la educación superior se constituye en un factor fundamental del referido proceso.

\subsection{Enfoque de la sostenibilidad en el marco de la formación en la educación superior}

Las iniciativas impulsadas en un orden global para reafirmar la relevancia de la sostenibilidad han sido promovidas por organismos como la Organización de Naciones Unidas (1987, 1992, 2015), la OCDE (2015), la 
UNESCO (2006), el Banco Interamericano de Desarrollo, BID (2016). En el mismo orden, han de vincularse las IES, desde su rol formativo, el cual amerita ser fortalecido para transferir y destacar la importancia de la formación bajo los principios y dimensiones que rigen la sostenibilidad. De tal forma, es necesario fortalecer las acciones que se vinculan con el currículo y determinar las adaptaciones que permiten desde la transversalidad del conocimiento promover procesos de aprendizajes asociados con el tema, y como tal se constituya en factor distintivo en los procesos formativos de las IES.

Como determinante en la dinámica educativa, se plantea ¿cómo formar bajo el compromiso de y para la sostenibilidad?, a través de lo cual el ser humano se apropie de la responsabilidad y el futuro asertivo del desarrollo ambiental, económico y social. Es un precepto complejo, que se visualiza desde la especificidad de cada área de conocimiento y programas académicos ofertados por las IES, como es el caso de los programas de ciencias administrativas para asociarlos según lo expuesto por la UNESCO (s/f) en el informe Decenio de las Naciones Unidas de la Educación para el Desarrollo Sostenible 2005-2014, cuyo propósito es movilizar los recursos educativos del mundo, para crear un futuro más sostenible. En el mismo orden, la UNESCO (2015a) en el Programa de Acción Mundial de Educación para el Desarrollo Sostenible 2015-2019, reconoce como elemento clave la educación de calidad, facilitadora crucial del desarrollo sostenible. A su vez destaca el reconocimiento otorgado a la educación a través de los objetivos de desarrollo sostenible para la consecución de sus metas para 2030.

Según lo expuesto, el proceso formativo para el caso de las ciencias administrativas, amerita apropiarse de los enfoques que permiten afianzar la formación con sentido del hacer hacia la sostenibilidad. De tal forma, que constituye una prioridad ser considerada en el marco de las reformas curriculares del sistema educativo superior. Para su contextualización se parte de los ejes temáticos y asignaturas de formación básica y profesional del área del conocimiento, hasta proyectar futuros modelajes en entornos organizacionales, constituyendo la adopción de prácticas pedagógicas y didácticas para soportar la formación en sostenibilidad un tema escasamente estudiado.

Sin embargo, asociar los resultados de lo expuesto con tendencias vinculadas con la gestión de la competitividad, se manifiesta a través de los indicadores estudiados promovidos por organismos internacionales, como el Foro Económico Mundial por sus siglas WEF (WEF, 2017) para identificar cuál es el direccionamiento que desde diferentes sectores como el educativo, económico y productivo, amerita considerarse para adoptar prácticas para afianzar la competitividad y sus implicaciones en el entorno laboral. (Cala et al. 2018) exponen "en la educación superior, gran parte del currículo, se concibe para contribuir con el proyecto de desarrollo y avance de una nación, así como también de mantener la cohesión social alrededor de las relaciones de poder particulares", p.3.

Al respecto, la orientación hacia diseños curriculares renovados, amerita que sean reestructurados otorgando prioridades en la formación en principios de la sostenibilidad, entre las demandas están responder al dinamismo de las sociedades, mercados y organizaciones, a través de las cuales, no solo han de preverse los procesos productivos (bienes y servicios), sino también el consumo responsable y la responsabilidad extendida del productor, entendida esta, como la capacidad del mismo para manejar los medios y recursos, así una vez finalizado el ciclo de vida de un producto, el usuario y la organización puedan dar vida útil a este.

En tal sentido, se requiere identificar, los riesgos y oportunidades que surgen de un entorno global, como también concebir un portafolio de acciones, mediadas por la docencia, investigación y proyección social, así como una articulada y eficiente gestión institucional que responda a los principios que rigen la sostenibilidad. Solis (2019) define la sostenibilidad desde un concepto abierto y flexible, que se ha ido moldeando a lo largo del tiempo, especialmente desde las tres últimas décadas en el que viene jugando un papel relevante en el debate socio-científico. De tal forma, la pertinencia de los contenidos: enfoques teóricos y experienciales, además de las 
tendencias pedagógicas, didácticas, como la infraestructura integrada por: docentes-investigadores, tecnologías y los contextos de intervención: sociedad-organizaciones se constituyen como base para la formación de profesionales, cuyas competencias determinen nuevas formas de impulsar y generar valor en pro de la sostenibilidad.

Las mencionadas competencias, se afianzan a partir de la necesidad de responder o promover sociedades más humanizadas y para enfrentar e interactuar de forma asertiva ante las dinámicas promovidas en un entorno global. Para viabilizar lo descrito, se parte de los desafíos de la educación superior impulsados por el Foro Mundial sobre la Educación, (UNESCO 2015b), la definición de estrategias de competencias para construir un futuro mejor (OCDE 2019), estrategias para la educación del desarrollo sostenible (UNECE) (2016), Novo (2006) con su enfoque hacia dónde queremos ir: el desarrollo sostenible.

En el mismo orden, la adopción del diseño curricular bajo esquemas como el disruptivo, (Pilonieta, 2017) significa comprender que es necesario superar la actual educación, para hacerla diferente, con el fin de responder a las exigencias del futuro, y encontrar los recursos necesarios para descubrir nuevos horizontes, dimensiones como escenarios, instrumentos y técnicas que contribuyan con el desarrollo académico, como humano considerado por el Programa de Naciones Unidas (2016).

El enfoque disruptivo, se plantea para el presente estudio, como la visión que ha de permitir enfrentar las dinámicas del siglo XXI, surgidas desde el entorno global: tecnologías, transformaciones socioeconómicas, competitividad de mercados, cultura e impulso hacia una nueva era de negocios. Según Pedroza (2018), este contribuye a promover conocimientos transdisciplinares, que trasciende fronteras y barreras, por lo que la formación desde el desarrollo del pensamiento complejo, constituye la base de la transformación curricular que se impulsa.

Entre los actores que lo viabilizan, se destacan los vinculados directamente con el proceso: sociedad-estudiantesdocentes, cuyos intereses académicos, ameritan estar reflejados en el currículo, esto a partir de la adaptación de currículos rígidos, semiflexibles y flexibles, a currículos interactivos. Por su parte, Fung (2017) propone el currículo conectado, propiciando una relación de mayor conexión entre estudiantes, investigadores y el mundo real, desde una visión interdisciplinaria que se afianza en el contexto de las instituciones de educación superior.

\section{Metodología}

A partir de una investigación básica, de tipo analítica- propositiva (McMillan y Schumacher, 2012), se desarrolla el enfoque metodológico, mediante el cual, se orienta una estructura sistémica, sobre las diferentes etapas que contienen el desarrollo de la reforma curricular en ciencias administrativas. Su enfoque prevé la interrelación de las funciones sustantivas de las IES, así como también los indicadores que soportan las variables: reforma curricular y formación para la sostenibilidad de profesionales en el área cuyo alcance se proyecta en las instituciones de educación superior en un orden global.

Para tal efecto, se procedió con la declaración de las interrogantes, (ver tabla1) basados en los indicadores que direccionaron el desarrollo de contenido de las variables a partir del contexto de estudio.

Desde un diseño no experimental, según (Marín-González et al. 2018); McMillan y Schumacher (2012), se llevó a efecto el estudio de las variables e indicadores, tal cual se presentan en el contexto de estudio seleccionado, respetando el entorno natural y de los agentes intervinientes en la búsqueda de las opiniones, valoraciones y actitudes de los participantes.

Bajo un paradigma cualitativo, se procedió con el diseño de matrices de análisis mediante las cuales se presenta la estructura interrelacionada de cada una de las etapas que soportan la disertación de la propuesta, cuya base 
permitió la representación del enfoque sistémico de los componentes que soportan la reforma curricular, según se explica.

Tabla 1

Interrogantes que soportan el objetivo del estudio

\begin{tabular}{cr}
\hline Literal & Interrogantes \\
\hline (A) & ¿Cuáles son las variables que justifican la reforma curricular?; \\
\hline (b) & ¿Cuál es el perfil del estudiante en ciencias administrativas?; \\
\hline (C) & ¿Cuáles son los resultados de las prácticas pedagógicas y didácticas aplicadas previas a la transformación? \\
\hline (D) & ¿Cuáles son las políticas de las IES, que promueven la formación continua, la investigación, el desarrollo \\
creativo e innovador y la movilidad de docentes-investigadores y estudiantes?
\end{tabular}

Fuente: elaboración propia

\subsection{Primera etapa: identificación del tipo de inviestigación}

Se fundamenta en un tipo de investigación analítica- propositiva (McMillan y Schumacher, 2012), a partir de la cual se logró el estudio de las variables trasformación curricular y formación para la sostenibilidad. Estas se conciben, como parte de la gestión que define la evolución y dinamización de la educación superior, que se demanda ante el contexto global.

La adopción del referido proceso se plantea al estructurarse las prácticas académicas, investigativas, y de proyección social, previendo su alcance teórico-práctico durante la formación profesional, en concordancia con la naturaleza y perfil de los sectores sociales y productivos en los cuales han de interactuar como profesionales en el ejercicio de las ciencias administrativas. Proceso que requiere de la identificación de los componentes asociados de forma sistémica, como soporte para afianzar la formación en el área.

Como estrategia que prevé la viabilidad de lo antes descrito, la iniciativa central está determinada por el fortalecimiento de la capacidad profesional, entre las prioridades están: (a) la pertinencia del enfoque curricular (b) la capacidad para generar la transformación del talento humano; (c) la concepción de competencias y capacidades permanentes que se fundamentan en la pedagogía y didáctica, cuya efectividad genera la plataforma que impulsa la competitividad profesional ante una sociedad disruptiva que promueve el cambio.

\subsection{Segunda etapa: Selección de la muestra}

Para el desarrollo analítico propositivo, la muestra según Hernández, et al. (2014) es considerada como la representación parcial o el subconjunto de unidades de análisis seleccionados de una población, mediante la cual se obtienen datos e información sobre un fenómeno que se estudia. Para el estudio, se aplicó una muestra no probabilística aleatoria, (McMillan y Schumacher (2012). Desde la perspectiva analítica documental, la muestra estuvo seleccionada mediante las fuentes de información documentada de tipo indexada, disponible en bases de datos digitales de alcance global. Proceso que permitió, su efectiva selección a partir de los criterios, que desde la perspectiva de este estudio garantiza la obtención de resultados pertinentes y con validez (ver tabla 2). 
Tabla 2

Distribución de la selección de la muestra

Naturaleza de la muestra

Criterios y alcance para la ejecución

seleccionada

\begin{tabular}{|c|c|}
\hline $\begin{array}{l}\text { Fuentes de la información: } \\
\text { impresa y digital. (Basada en } \\
\text { la rigurosidad de la } \\
\text { selección) }\end{array}$ & $\begin{array}{l}\text { - Revisión y selección de Artículos Científicos disponibles en bases de datos } \\
\text { indexadas de alcance nacional e internacional. } \\
\text { - Validación de perfiles académicos de egreso del profesional de Administración } \\
\text { disponibles en páginas web de orden nacional e internacional. } \\
\text { - Análisis sobre la epistemología, importancia y tendencias en las ciencias } \\
\text { administrativas. }\end{array}$ \\
\hline $\begin{array}{l}\text { Conformación de grupos } \\
\text { focales }\end{array}$ & $\begin{array}{l}\text { - Difusión sobre el por qué de la transformación curricular. } \\
\text { Interacción de egresados, estudiantes, docentes e investigadores. }\end{array}$ \\
\hline $\begin{array}{l}\text { Conformación de equipos de } \\
\text { alto desempeño. }\end{array}$ & $\begin{array}{l}\text { - Análisis y aportes ante tendencias y desarrollos en el área de conocimiento, cuyos } \\
\text { enfoques soportan la proyección del modelo curricular. } \\
\text { - Interacción con Consultores, directivos de universidades, y empresarios de orden } \\
\text { internacional. }\end{array}$ \\
\hline $\begin{array}{l}\text { Participación de Expertos - } \\
\text { Programas formativos. }\end{array}$ & $\begin{array}{l}\text { - Interacción sobre expectativas, avances y dinámicas que promueven la reforma de } \\
\text { la educación superior. Impulso al Coworking, para el logro de objetivos académicos } \\
\text { que determinan el desarrollo profesional. } \\
\text { - Programación de talleres de interacción con docentes e investigadores. }\end{array}$ \\
\hline Designación comité curricular & $\begin{array}{l}\text { - Estudio sobre estructuras programáticas, compartir y tomar decisiones para } \\
\text { aprobar estructura, pilares, enfoques y tendencias con las cuales se alinea y se } \\
\text { pretende responder hacia la formación pertinente de un profesional en ciencias } \\
\text { administrativas. }\end{array}$ \\
\hline Designación comité ampliado & $\begin{array}{l}\text { - Socialización sobre la justificación de la transformación. } \\
\text { - Disertación sobre posibles efectos de la reforma curricular ante demandas del } \\
\text { entorno, docentes, investigadores, y estudiantes. } \\
\text { - Interacción sobre capacidades para generar aportes a partir de la matriz curricular } \\
\text { general. } \\
\text { - Generación de aportes centrados en el análisis, interrelación y vinculación de las } \\
\text { áreas de la formación: a) básica general; (b) básica profesional; y (c) profesional } \\
\text { específica; en el marco de las competencias que ameritan ser desarrolladas durante } \\
\text { la formación profesional. }\end{array}$ \\
\hline
\end{tabular}

Fuente: elaboración propia.

\subsection{Tercera etapa: adopción de enfoques o paradigmas que orientan la reforma}

La adopción del enfoque metodológico que contribuye con la viabilidad y rigurosidad de la reforma curricular, está precedida por un enfoque cualitativo, cuya aplicación permitió el tratamiento de la información obtenida, a través de los criterios definidos para la selección de la muestra previamente identificada. La relevancia de su aplicación, se concibe desde una perspectiva integral para proyectar una visión más real sobre las transformaciones y avances de la educación superior surgidas en la última década.

La rigurosidad sobre prácticas que se han de considerar durante el proceso de la reforma curricular, le otorgan validez siempre y cuando el enfoque cualitativo se conciba integrando con factores cuantitativos según se describe en tabla 3. La adopción de ambos enfoques, parte de la capacidad de proyectarlos desde lo teórico y lo práctico. En consecuencia, su viabilidad, permite afianzar la capacidad de una reforma curricular asertiva, y orientada en beneficio de la pertinencia de una formación direccionada a la ejecución de acciones que redundan en la sostenibilidad y por ende resalta los principios de responsabilidad social de las IES, para con las regiones. 
Tabla 3

Enfoques estratégicos para la viabilidad de la reforma curricular

\begin{tabular}{|c|c|c|}
\hline Enfoques & Alcance & Componentes intervinientes \\
\hline Cualitativo & $\begin{array}{l}\text { Alineado en función de los principios rectores de las } \\
\text { IES. Su enfoque central parte de la naturaleza y } \\
\text { razón de ser, y se complementa con el enfoque que } \\
\text { rige la estructura organizacional académica, la } \\
\text { cultura, la concepción de un sistema fortalecido con } \\
\text { respecto a la academia, investigación y extensión. }\end{array}$ & $\begin{array}{l}\text { - Principios de gestión. } \\
\text { - Portafolio de programas académicos } \\
\text { - Organigrama estructural. } \\
\text { - Alcance de las funciones sustantivas. }\end{array}$ \\
\hline Cuantitativo & $\begin{array}{l}\text { Identifica y responde al cumplimiento de indicadores } \\
\text { a partir del alcance de la infraestructura técnica- } \\
\text { operativa para promover los estándares de calidad } \\
\text { que miden la eficiencia de los recursos económicos, } \\
\text { la cualificación y perfeccionamiento continuo del } \\
\text { activo humano, optimización de los recursos } \\
\text { tecnológicos como estrategia para la transferencia, } \\
\text { socialización de conocimiento e inteligencia } \\
\text { institucional, así como la dinamización de los } \\
\text { procesos que respaldan las funciones sustantivas de } \\
\text { docencia, investigación, extensión y gestión } \\
\text { administrativa. }\end{array}$ & $\begin{array}{l}\text { - Capacidades de la infraestructura: } \\
\text { determinada desde los centros, institutos, } \\
\text { laboratorios. } \\
\text { - Inversiones- Retorno. } \\
\text { - Plataforma de docentes, investigadores, } \\
\text { estudiantes, personal técnico, } \\
\text { comunidades y sectores productivos } \\
\text { vinculados. } \\
\text { - Plataformas tecnológicas. } \\
\text { - Efectividad de los estándares de procesos } \\
\text { y cumplimiento de objetivos académicos- } \\
\text { investigativos de proyección social y } \\
\text { administrativos. }\end{array}$ \\
\hline
\end{tabular}

Fuente: elaboración propia

El alcance y la identificación de los componentes intervinientes en los enfoques mencionados, crean las bases para generar los principios que soportan cada una de las etapas de la metodología descrita. A través de esta, se logra alinear la gestión que soporta la reforma curricular, y por ende alcanzar objetivos formativos y de mejoramiento continuo que impulsa el desarrollo profesional en las ciencias administrativas, con impacto social.

\subsection{Cuarta etapa: técnicas e instrumentos}

Fundamentar el desarrollo efectivo de las prácticas que viabilizan la reforma curricular, se vincula con la selección asertiva de técnicas e instrumentos. De esta forma, se garantiza obtener información bajo estándares de validez y pertinencia, para lograr una aproximación real sobre las dinámicas surgidas en la educación superior en el siglo $\mathrm{XXI}$, las cuales están precedidas por la ya reconocida transformación de orden global en la cual interviene de forma directa la denominada revolución 4.0.

Pedroza (2018) concibe los efectos de esta revolución en el contexto educativo, a partir de la incidencia de las tecnologías del aprendizaje. Entre los efectos principales esta la diversificación sobre formas de aprendizajes: en línea, el móvil, la gamificación y la inteligencia de datos, acompañados de laboratorios virtuales, laboratorios de neuroaprendizaje digitales, simulaciones holográficas, presentaciones en séptima dimensión (7D) y redes de colaboración tecnológica.

Sumado a lo anterior, se consideran las técnicas principales para el diseño curricular, y su alcance necesario en el antes, durante y posterior del proceso formativo. Entre las principales estrategias didácticas y de apoyo como instrumentos para su desarrollo se destacan: a) la prospectiva: método Delphi, técnica de escenarios, grupos focales; b) encuesta: fundamentada mediante el diseño de cuestionarios; c) análisis documental: soportado por 
diseño de matrices de relacionamiento teórico; d) técnicas estadísticas: descriptivas, multivariables. El alcance de estas, varía a partir del direccionamiento que cada IES le otorgue al proceso.

\subsection{Quinta etapa: análisis y contextualización de la propuesta}

Parte de la consolidación del enfoque sistémico, el cual se fundamenta mediante una metodología integradora y de interrelación, que permite la transversalidad de todos los componentes que viabilizan la reforma curricular que se consideran formar para la sostenibilidad: a) docencia: vigencia de programas académicos; b) investigación: como eje transversal concebido desde el inicio de la formación profesional en las ciencias administrativas y sus implicaciones sobre la sostenibilidad en los contextos Universidad-Empresa-EstadoSociedad, bases para la viabilidad del desarrollo sostenible en lo económico, social y ambiental, c) proyección social: capacidad de articulación y d) gestión institucional.

\section{Resultados}

A partir de las etapas descritas en el enfoque metodológico, las cuales fueron analizadas considerando: 1) la interrelación de los fundamentaos teóricos (estudio documentado) y 2) el desarrollo aplicativo surgido desde la perspectiva experiencial en IES, se fundamenta el enfoque sistémico en figura 1, cuyo desarrollo facilitó representar las diferentes etapas que permiten argumentar su viabilidad.

La visión integral de las etapas presentadas desde una visión sistémica, prevé impulsar la dinamización del proceso que rige la reforma curricular. Esta se representa, a partir de la descripción de cada componente que soporta el proceso, y a su vez, contribuye a orientar las acciones considerando los diferentes indicadores que determinan el alcance de cada función sustantiva que rige la educación superior.

Con el fin de afianzar la transversalidad de la formación en sostenibilidad, desde las ciencias administrativas, con proyección hacia otras áreas de conocimiento, se expone los factores que justifican la interrelación de las variables:

a) Necesidad de afianzar desde un entorno global, acciones que contribuyan desde las diferentes áreas de conocimiento el ejercico de prácticas de sostenibilidad en la actualidad con previsión hacia el futuro.

b) Incremento de los estándares que regulan la competitividad de los contextos en los cuales intervienen los profesionales de las ciencias administrativas.

c) Renovación y actualización de las áreas de conocimiento disciplinar vinculado con las ciencias administrativas y áreas de conocimiento asociadas.

d) Transformaciones sociales que requieren de la renovación de las IES, sumado a la capacidad de estas para configurar la estructura del currículo en el marco del desarrollo de competencias humanas, cognitivas y técnicas procedimentales, que redunden en el beneficio de entornos sociales activos.

e) Proyección del modelo de formación continua de docentes, como base que proyecta el éxito y viabilidad de la trasformación curricular.

f) Un nuevo enfoque centrado en la dinamización de la gestión de investigación, como eje que contribuye con la consolidación de competencias para el ejercicio profesional competitivo, siempre y cuando sus prácticas se viabilicen desde la transversalidad de las áreas y procesos formativos.

g) Fortalecimiento al impulso de la creatividad, innovación y emprendimiento como medio que promueve el trabajo independiente y el desarrollo socio productivo que minimice el impacto que vulnera el entorno global.

h) Consolidación de infraestructuras que fortalezcan las capacidades del desarrollo que afianza el proceso formativo: docentes, centros, laboratorios, institutos, tecnologías de información y comunicación, 
renovación de prácticas pedagógicas y didácticas, estrategias de movilidad estudiantil de docentes e investigadores, con el fin de analizar entornos desde un marco de intervención complejo y diverso.

i) La interrelación del proceso, prevé garantizar la estandarización de los sistemas y principios que rigen la calidad educativa superior, como proyección de soporte a la gestión de acreditación de los programas.

Figura 1

Enfoque sistémico de la interrelación de resultados

de las etapas que soportan la reforma curricular.

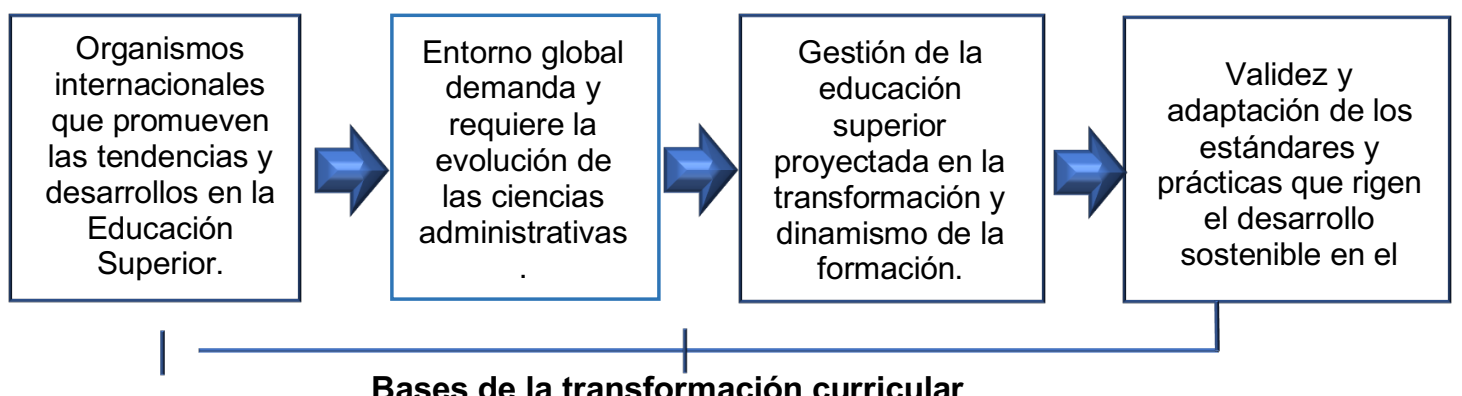

Bases de la transformación curricular

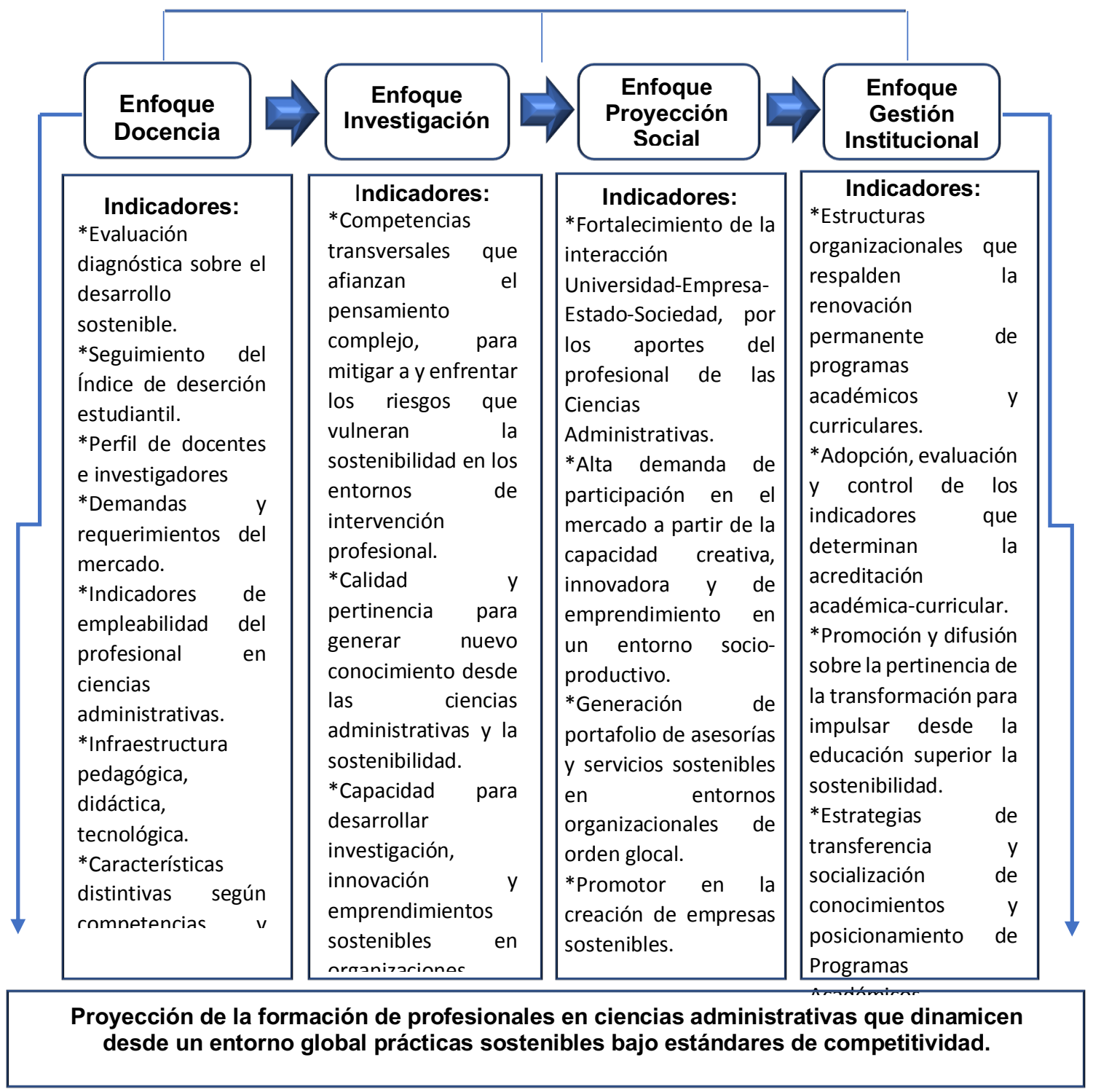

Fuente: elaboración propia. 
Entre la proyección de la propuesta de las etapas descritas, León, et al. (2014), plantean la importancia de las estrategias didácticas, utilizadas por los educandos en sus carreras. Valderrama, et al. (2020) plantea la aplicación de la metodología basada en el aprendizaje-servicio, como metodología que permite involucrar al estudiante en proyectos con impacto social o ambiental. También se apunta al uso de diarios como recursos de aprendizaje para reflexionar y sensibilizar sobre la sostenibilidad. Sin embargo, en el portafolio de opciones se destaca, la metodología basada en retos y problemas, las cuales se conciben con referentes para generar a partir de la apropiación del conocimiento, soluciones ante los riesgos e incertidumbres que vulneran las regiones en materia de sostenibilidad.

Tal concepción, contribuye además de viabilizar reformas educativas, consolidar un sistema educativo disruptivo, capaz de convertirse en referente sobre métodos de viabilizar transformaciones curriculares bajo un enfoque valido, medible y confiable, en el ámbito de la educación superior que se proyecta en entornos socio-organizacionales. Como tal, amerita ser visualizado para prever desde los escenarios actuales y futuros la capacidad de participación de los profesionales en ciencias administrativas en entornos organizacionales públicos y privados.

\section{Conclusiones}

Sistematizar las etapas de la reforma curricular en los programas de ciencias administrativas para la formación en sostenibilidad, permitió concluir sobre la importancia de comprender el alcance de cada una de las etapas que fundamentan la interrelación de los diferentes procesos que viabilizan la reforma curicular, cuyo viabilidad ha sido concebida desde una visión documentada-teoría y la práctica desde el contexto de las IES.

Planteamiento que contribuye a dar respuesta a lo expuesto por Halloran, y Friday, (2018) en su estudio sobre: ¿Pueden las universidades de hoy liderar el aprendizaje para el mañana? La universidad del futuro, para lo cual conciben los principales focos de desarrollos: (a) tecnología (cambios exponenciales); (b) la geopolítica (entornos volátiles, inciertos, complejos y ambiguos); (c) la economía (dinámica); y (d) el medio ambiente (cambio climático).

Focos de desarrollos que en el siglo XXI demandan de una nueva mirada por profesionales, en el caso de los egresados en ciencias administrativas, cuya formación transversal basada en la sostenibilidad, amerita ser fortalecida y precedida por los estándares de competitividad que promueven las dimensiones: social, económica y ambiental. Asimismo, la sistematización presentada se orienta en las capacidades de apropiación de procesos formativos en los que prevalezca el aprendizaje y competencias centradas en el análisis, la interpretación, cocreación, investigación innovación, y emprendimiento mediado por las tecnologías, acordes con la evolución de las ciencias administrativas que den respuestas a las exigencias del mercado global en el marco de las sociedades disruptivas y emergentes.

A través de lo expuesto, se genera un espacio para la disertación y desarrollo de futuras investigaciones, que permitan fortalecer la línea temática sobre educación-reforma curricular-formación para la sostenibilidadevaluación, las cuales son variables que ameritan ser complementadas a partir de los constructos expuestos, en los que prevalezca la sostenibilidad asociada con: a) la pertinencia académica; b) procesos de formación bajo principios de integralidad; c) la rigurosidad científica y experiencial; d) la inclusión; e) diversidad de género; y e) la pluriculturalidad, constituyen parte de la gestión educativa con pertinencia hacia el desarrollo social, económico y productivo.

\section{Declaración de intereses}

Las autoras afirman no existir conflictos de intereses. 


\section{Referencias bibliográficas}

Banco Interamericano de Desarrollo (2016): Informe de Sostenibilidad del BID 2016. Disponible en: https://publications.iadb.org/publications/spanish/document/Informe-de-Sostenibilidad-del-BID-2016.pdf

Brunner, Dr. J.J., \& Pedraja-Rejas, Dra. Liliana. (2017). Los desafíos de la gobernanza de la educación superior en Iberoamérica. Ingeniare. Revista chilena de ingeniería, 25(1), 2-7. https://dx.doi.org/10.4067/S071833052017000100002

Cala, Verónica C.; Soriano-Ayala, Encarnación \& López-Martínez Manuel J. (2018). Actitudes hacia personas refugiadas y ciudadanía europea inclusiva. Análisis para una propuesta educativa intercultural con el profesorado en formación. RELIEVE, 24(2), art. M1. doi: http://doi.org/10.7203/relieve.24.2.13374

Fung, D. (2017). A connected Curriculum for Higher Education. London, England: UCL Press. Disponible en: https://discovery.ucl.ac.uk/id/eprint/1558776/1/A-Connected-Curriculum-for-Higher-Education.pdf

Halloran, Lucille, y Friday, Catherine (2018) Can the universities of today lead learning for tomorrow? The University of the Future. Ernst \& Young Buiding a Better Working World. Disponible en: https://assets.ey.com/content/dam/ey-sites/ey-com/en_au/topics/government-and-public-sector/eyuniversity-of-the-future-2030.pdf

Hernández Sampieri, R., Fernández Collado, C. \&amp; Baptista Lucio, P. (2014). Metodología de la investigación. (6a Ed.) Mc Graw Hill Education.

León Urquijo, A., Risco del Valle, E. y Alarcón Salvo, C. (2014). Estrategias de aprendizaje en educación superior en un modelo curricular por competencias. Revista de la Educación Superior Vol. xliii (4); No. 172, octubre-diciembre del 2014. ISSN: 0185-2760. (p. 123-144). Disponible en: http://www.scielo.org.mx/pdf/resu/v43n172/v43n172a7.pdf

Marín-González, F., Cabas, L. de J., Cabas, L. C., \& Paredes-Chacín, A. J. (2018). Formación Integral en Profesionales de la Ingeniería. Análisis en el Plano de la Calidad Educativa. Formación Universitaria, 11(1), 13-24. doi:10.4067/s0718-50062018000100013

McMillan-Sally, J., y Schumacher, S. (2012). Fundamentos y Metodología de Investigación en educación II. México. PEARSON. P.397.

Muñoz Portillo, J. (2017). Política en las políticas de calidad de la educación en Centroamérica. América Latina Hoy, 77,115-136. Consultado: 20.7.20. Disponible en: https://www.redalyc.org/articulo.oa?id=308/30854700006

Naciones Unidas; ONU. (1987). Informe Brundtland, 1987 de la Comisión Mundial para le Medio Ambiente y el Desarrollo. Disponible en: http://www.ecominga.uqam.ca/PDF/BIBLIOGRAPHIE/GUIDE_LECTURE_1/CMMAD-Informe-ComisionBrundtland-sobre-Medio-Ambiente-Desarrollo.pdf

Naciones Unidas; ONU. (1992) Agenda 21. United Nations Conference on Environment and Development, Río de Janeiro. Disponible en: https://www.un.org/spanish/esa/sustdev/agenda21/index.htm

Naciones Unidas; ONU. (2015). Objetivos de Desarrollo Sostenible. Disponible en: https://www.un.org/sustainabledevelopment/es/objetivos-de-desarrollo- sostenible/ 
Naciones Unidas para la Educación, la Ciencia y la Cultura. UNESCO. (2006). Directrices en materia de calidad de la educación superior a través de las fronteras. Paris. Disponible en:

http://www.unesco.org/education/hed/guidelines

Novo, M. (2006) El desarrollo sostenible. Su dimensión ambiental y educativa, 431 pp. Madrid: Pearson/UNESCO.

Oficina Regional de Educación para América Latina y el Caribe. OREALC/UNESCO Santiago. (2007). Educación de calidad para todos: un asunto de derechos humanos. Documento de discusión sobre políticas educativas en el marco de la II Reunión Intergubernamental del Proyecto Regional de Educación para América Latina y el Caribe (EPT/PRELAC). Santiago de Chile: OREALC/UNESCO-Santiago.

Organisation for Economic Co-operation and Development OCDE (1996). The Knowledge-Based Economy. OCDE/GD (96)102. París. Disponible:

http://www.oecd.org/officialdocuments/publicdisplaydocumentpdf/?cote=OCDE/GD\%2896\%29102\&docL anguage $=\mathrm{En}$

OCDE. (2019). Estrategia de Competencias de la OCDE 2019. Competencias para construir un futuro mejor. Disponible en: https://www.oecd.org/publications/estrategia-de-competencias-de-la-ocde-2019e3527cfb-es.htm

Pedroza Flores, R. (2018). La Universidad 4.0 con currículo inteligente 1.0 en la cuarta revolución industrial. Revista Iberoamericana para la Investigación y el Desarrollo Educativo, Vol. 9, no. 17.

Pilonieta, G. (2017). Innovación disruptiva. Esperanza para la educación futura. Revista Educación y Ciudad, (32), 53-64. doi: 10.36737 / 01230425.Vo.n32.2017.1627.

Programa de las Naciones Unidas para el Desarrollo PNUD.(2016). Informe sobre Desarrollo Humano 2016. Oficina del Informe sobre Desarrollo Humano (OIDH). Disponible en: https://www.undp.org/content/dam/undp/library/corporate/HDR/HDR2016/HDR16\%200verview\%20Spa nish.pdf

Qu, Dongxu, Shevchenko, T., \& Yan, X. (2020). University Curriculum Education Activities Towards Circular Economy Implementation. International Journal Of Scientific \& Technology Research. Vol. 9. Disponible en: http://www.ijstr.org/final- print/may2020/University-Curriculum-Education-Activities-TowardsCircular-Economy-Implementation.pdf

Ratcliff, J.L., What is a curriculum and what it should be?, in Gaff, J.G. \& Ratcliff J.L. (eds.) (1997). Handbook of the Undergraduate Curriculum. A Comprehensive Guide to Purposes, Structures, Practices and Change, Jossey-Bass Pub., San Francisco.

Silva Montes, C. (2015). La reforma curricular en competencias: La experiencia en las preparatorias federales por cooperación. Archivos Analíticos de Políticas Educativas, 23(68).

http://dx.doi.org/10.14507/epaa.v23.1727

Solís-Espallargas, C. (2019). La percepción de la sostenibilidad de estudiantes de Máster en educación ante los objetivos de desarrollo sostenible. Revista ESPACIOS. ISSN 07981015 Vol. 40 (№ 39). Disponible en: https://www.revistaespacios.com/a19v40n39/a19v40n39p11.pdf

The World Economic Forum. WEF. (2017). The global competitiveness report: 2017- 2018. New York, USA: World Economic Forum. Disponible: https://www.weforum.org/reports/the-global-competitivenessreport-2017-2018 
UNESCO. (2008). Reflexiones en torno a la evaluación de la calidad educativa en América Latina y el Caribe. Laboratorio latinoamericano de evaluación de la calidad en educación. Disponible en: https://unesdoc.unesco.org/ark:/48223/pf0000177648

UNESCO. (2009). Conferencia mundial sobre la educación superior. La nueva dinámica de la educación superior y la investigación para el cambio social y el desarrollo. disponible: Revista Da Avaliação Da Educação Superior (Campinas), 14(3), 755-766. doi: 10.1590/s1414-40772009000300013.

UNESCO. (2015a) Programa de acción mundial para la Educación para el Desarrollo Sostenible (2015-2019). Disponible en: https://es.unesco.org/gap

UNESCO. (2015b) Educación 2030: Declaración de Incheon y marco de acción para la realización del Objetivo de Desarrollo Sostenible 4. Disponible en: https://www.buenosaires.iiep.unesco.org/es/publicaciones/educacion-2030-declaracion-de-incheon-ymarco-de-accion

UNESCO. (s/f) El Decenio de las Naciones Unidas para la EDS 2005-2014.

Disponible:https://es.unesco.org/themes/educacion-desarrollo-sostenible/comprender-EDS/decenio-onu

United Nations Economic Commission For Europe (UNECE) (2016). Ten years of the UNECE Strategy for Education for Sustainable Development. Nueva York y Ginebra. Disponible en: http://www.unece.org/fileadmin/DAM/env/esd/ESD_Publications/10_years_UNECE_Strategy_for_ESD.pd f

Valderrama-Hernández, R.; Alcántara Rubio, L.; Sánchez-Carracedo, F.; Caballero, D.; Serrate, S.; Gil-Doménech, D.; Vidal-Raméntol, S., y Miñano, R. (2020). ¿Forma en sostenibilidad el sistema universitario español? Visión del alumnado de cuatro universidades. Educación XX1, 23(1), 221-245, doi: 10.5944/educXX1.2342 